\title{
Tooth survival after root canal treatment
}

\section{Abstracted from \\ Ng YL, Mann V, Gulabivala K.}

Tooth survival following non-surgical root canal treatment: a systematic review of the literature. Int Endod J 2010; 43: 171-189.

Address for correspondence: Y-L Ng, Unit of Endodontology, UCL Eastman Dental Institute, UCL, 256 Grays Inn Road, London WC1X 8LD, UK.

E-mail: p.ng@eastman.ucl.ac.uk

\section{Question: What factors affect tooth survival following non-surgical root canal treatment $(\mathrm{RCTx})$ ?}

Data sources Medline, the Cochrane Library, hand searches of the International Endodontic Journal, Journal of Endodontics, Oral Surgery Oral Medicine Oral Pathology Oral Radiology and Endodontics, Dental Traumatology (\& Endodontics) and bibliographies of all relevant articles and review articles. Unpublished studies were identified by searching abstracts and conference proceedings. Personal contacts were used to identify ongoing or unpublished studies. Two reviewers independently assessed and selected the studies with disagreements being resolved by discussion.

Study selection Clinical studies of RCTx on more than 30 teeth and of at least six-month duration, where the success was based on survival of tooth and the proportion of teeth surviving was given, or could be calculated from the raw data, were included.

Data extraction and synthesis Data were extracted by two reviewers independently using custom-designed forms. The weighted pooled proportion of teeth surviving after treatment and the combined effects (expressed as odds ratio) of clinical factors on tooth survival were estimated using fixed and random effects meta-analyses using DerSimonean and Laird's methods. The survival data were pooled into three groups based on the duration after treatment: 2 or 3 years; 4 or 5 years; and 8, 9 or 10 years. Statistical heterogeneity amongst the studies was assessed by Cochran's (Q) test.

Results Of the 31 articles identified, 14 studies were included. The majority (10) were retrospective. The reported survival is shown in Table 1. Substantial differences in study characteristics were found to hinder effective direct comparison of findings. Evidence for the effect of prognostic factors on tooth survival was weak. Based on the data available for meta-analysis, four conditions were found to significantly improve tooth survival. In descending order of influence, the

Table 1. The pooled percentages of reported tooth survival

\begin{tabular}{l|l|l}
\hline Follow up period & mean & $95 \% \mathrm{Cl}$ \\
\hline $2-3$ years & $86 \%$ & $75-98 \%$ \\
\hline $4-5$ years & $93 \%$ & $92-94 \%$ \\
\hline $8-10$ years & $87 \%$ & $82-92 \%$
\end{tabular}

conditions increasing observed proportion of survival were as follows: (i) a crown restoration after RCTx; (ii) tooth having both mesial and distal proximal contacts; (iii) tooth not functioning as an abutment for removable or fixed prosthesis; and (iv) tooth type or specifically non-molar teeth. Statistical heterogeneity was substantial in some cases, but its source could not be investigated because of insufficient available information.

Conclusions The pooled proportion of teeth surviving over 2-10 years following RCTx ranged between $86 \%$ and $93 \%$. Four factors (listed above) were identified as significant prognostic factors with concurrence between all three strands of evidence.

\section{Commentary}

The literature is replete with studies on the outcome of non-surgical root canal treatment, which has been reported to have a success rate of 53-95\% over various periods of observation. ${ }^{1}$ When reviewing these studies, it quickly becomes apparent that outcome definitions and classification have been inconsistent. This, in turn has resulted in considerable variability of the reported 'success' rates. Having said that, this report presents a rather objective quantification of outcome of primary endodontic treatment, which is tooth survival. This evaluation method not only eliminates the tremendous subjectivity involved in trying to assess success and failure, but also makes way for a fair comparison with the survival rate of dental implants.

The eight prognostic factors mentioned in the study (qualification of operators, tooth type, remaining tooth structure, pre-operative pulpal and periapical status, apical extent of root filling, quality of root filling, time of coronal restoration, number of proximal contact) are the most relevant factors affecting the course of endodontic diseases. In light of the current understanding of the importance of creating a path for the irrigation solution to reach to the apex, apical width might be considered as a potential prognostic factors together with the irrigation methods.

However, endodontically treated tooth survival in this study has been found to be significantly associated with four conditions related to remaining tooth structure, post obturation treatment, occlusal status and one related to the complexity of anatomy and procedures which are molar teeth. These conditions are different from those reported in other studies, ${ }^{2}$ in which intrapulpal status and the presence of periapical pathologies were the most prominent when assessing success. Taken all together, it appears factors affecting tooth survival post endodontic treatment are different from those 
effecting the endodontic therapy. A cautious look at tooth survival however, should be undertaken with the emerging evidence relating oral foci of infection to systemic diseases.$^{3}$

\section{Practice points}

- Tooth survival after root canal treatment is high and comparable to intra-osseous fixtures.

- Preserving tooth structure is an important factor to be undertaken in the bio-engineering of coronal and radicular root canal preparation.

- Post endodontic treatment plans should precede the decisionmaking for root canal therapy.

- Excessive root canal preparations with greater taper files should be revisited in the light of the results of this study and other reports relating increasing vertical root fracture to excessive coronal pre-flaring and excessive apical width.

\section{Khaled Balto}

Department of Endodontics, King Abdulaziz University, Faculty of Dentistry, Saudi Arabia

1. Friedman S. Treatment outcome and prognosis of endodontic therapy. In Òrstavik D, Pitt-Ford TR (eds). Essential endodontology. pp 367-401. Malden, MA: Blackwell Science, 1998.

2. Òrstavik D. Time course and risk analyses of the development and healing of chronic apical periodontitis in man. Int Endod / 1996; 29: 150-155

3. Barnett B (2006). The oral-systemic disease connection. An update for the practicing dentist. J Am Dent Assoc; 137 (Suppl 2): 5S-6S.

Evidence-Based Dentistry (2011) 12, 10-11. doi:10.1038/sj.ebd.6400772

A short podcast overview of of this summary is available on the journal's website at http://www.nature.com/ebd/index.html 